\title{
Comment on: Deliberate Self-harm in adolescents
}

Singapore Med J 2015; 56(9): 530 doi: 10.11622/smedj.2015141

Dear Sir,

I am writing to add to the pertinent points raised by the article 'Deliberate self-harm in adolescents'. ${ }^{(1)}$ Beyond the role of primary care physicians and specialist care, parents and school counsellors can access a community-based mental health programme called REACH (Response, Early intervention and Assessment in Community mental Health) ${ }^{(2,3)}$ to help children and adolescents cope with self-harm behaviours. This programme is available to all students from mainstream and special schools in Singapore. The REACH team comprises different allied health professionals such as psychologists, occupational therapists, registered nurses and medical social workers in each of the four teams (in accordance with the Ministry of Education's school zoning). After obtaining important information on the students from the school counsellor and a detailed triaging process, REACH's multidisciplinary team responds in a timely manner based on the severity of the mental health condition. With its strategic location in the community and away from hospitals, REACH epitomises the term 'right-siting' of healthcare. In cases where the self-harm behaviour is due to a serious mental illness, REACH is able to quickly refer the student to a child psychiatrist clinic for further evaluation and management. With this approach, patients do not face the stigma of having to visit psychiatric clinics and there is less strain on the tertiary psychiatric clinics.

REACH was first developed in 2007 under the National Mental Health Blueprint and is now operated by three hospitals - the North and South teams are under the Institute of Mental Health, while the West and East teams are under the National University Health System and KK Women's and Children's Hospital, respectively. With the parents' consent, staff from the REACH team visit the students' school or home to conduct an assessment or intervention. As the first assessment is free of charge (subsequent interventions are chargeable) and interventions are conducted in school or at home, REACH's service is attractive to both parents and students. A case conference with the school and the student's parents is also conducted at the end of the assessment and before any interventions are started. REACH's service is meant to be time-limited with clear continuity-of-care guidelines and discharge policies.

REACH aims to administer secondary intervention while bringing mental health care closer to students. ${ }^{(4)}$ With general practitioners and voluntary welfare organisation staff trained in basic child mental health joining REACH as community partners, we hope that a growing mental health network will eventually be set up in Singapore to provide more seamless and effective mental health care for our child and adolescent populations.

Yours sincerely,

Say How $\underline{\text { Ong }^{1,2}}$

${ }^{1}$ Department of Child and Adolescent Psychiatry, Institute of Mental Health, ${ }^{2}$ Child and Adolescent Mental Wellness Service, Department of Psychological Medicine, KK Women's and Children's Hospital, Singapore. say_how_ong@imh.com.sg

\section{References}

1. Lauw M, How CH, Loh C. Deliberate self-harm in adolescents. Singapore Med J 2015; 56:306-9.

2. Xie Y, Boon J, Sim WH, Fung DA. Singapore model - REACH. In: Kutcher S, Wei Y, Veist MD, eds. School Mental Health: Global Challenges and Opportunities. Cambridge: Cambridge University Press, 2015: 202-17.

3. Fung D, Ong LP, Tay SL, Sim WH. REACH Chronicles: A Community Mental Health Model For Children and Adolescents in Singapore. Singapore: World Scientific Publishers, 2012.

4. Lim CG, Ong SH, Chin CH, Fung DS. Child and adolescent psychiatry services in Singapore. Child Adolesc Psychiatry Ment Health 2015 ; 9:7. 